\title{
Nanostructure of Thin Gold Films Investigated by Means of Atomic Force Microscopy and X-Ray Reflectometry Methods
}

\author{
D. ŻYMierskA ${ }^{a, *}$, J. Auleytner ${ }^{a}$, J. DomagatA ${ }^{a}$, \\ T. KOBIELA ${ }^{b}$ AND R. Duśb \\ ${ }^{a}$ Institute of Physics, Polish Academy of Sciences \\ al. Lotników 32/46, 02-668 Warsaw, Poland \\ ${ }^{b}$ Institute of Physical Chemistry, Polish Academy of Sciences \\ Kasprzaka 44/52, 01-224 Warsaw, Poland
}

\begin{abstract}
A study of the thin gold film growth, during the deposition on glass substrate under UHV conditions at low temperatures, is presented. The complementary methods, the atomic force microscopy and grazing incidence $\mathrm{X}$-ray reflectometry, are used for the research. It is shown that due to variation of the time of deposition from 2 to 50 min different kinds of thin $\mathrm{Au}$ films nanostructures are obtained: from discontinuous films consisting of isolated islands, via formation of the chains of islands, up to continuous films.
\end{abstract}

PACS numbers: 68.55.-a, 68.37.Ps, 61.10.Kw

\section{Introduction}

Gold is the unique element of high resistivity against surface reaction with the ambient atmosphere. For this reason thin gold films are widely used for construction of electrical junctions in microelectronic devices and solid-state chemical sensors. The structure of these electrical contacts is of crucial importance for their application. In the present work the structure of thin Au films deposited on glass at low temperature and only slightly sintered is studied. Up to now, majority of thin Au film research was carried out using high temperature deposition.

Physical properties of a thin metallic film on an insulating support strongly depend on the film morphology. In dependence on the material of the substrate and the deposited metal various structures of thin film can be obtained. The aim of

*corresponding author; e-mail: zymier@ifpan.edu.pl 
the paper is a research of the surface structure and morphology of thin gold films obtained under the same condition but different duration of deposition on glass. We carry out the investigations by means of two complementary methods, atomic force microscopy (AFM) and grazing incidence X-ray reflectometry (GIXR). The AFM allows one to determine a type and size distribution of inhomogeneities occurring in every stage of the film formation, while X-ray study gives an average roughness of the surface.

\section{Experimental}

\subsection{Thin Au film preparation}

Thin gold film deposition was carried out in an ultrahigh vacuum glass system routinely reaching $\approx 10^{-8} \mathrm{~Pa}$. Glass microscope plates from Menzel Glaser (Germany) were chosen as a substrate. The plates were placed on the bottom of the glass chamber. The construction of chamber allows one to measure the film resistance during the evaporation. All of the depositions were carried out having the chamber maintained at $78 \mathrm{~K}$. After the deposition, the films were annealed at $370 \mathrm{~K}$ for 20 min under UHV conditions. The different Au films were obtained by changing the time of evaporation. When 3 min exposure was applied the transparent pale purple films (labelled A) of the average thickness of about $5 \mathrm{~nm}$ were obtained. The deposition of 8 min gives the green transparent films (labelled B) of the average thickness below $20 \mathrm{~nm}$. After evaporation of $50 \mathrm{~min}$ non-transparent films (labelled C) of golden colour and of average thickness over $50 \mathrm{~nm}$ were obtained. All other evaporation parameters including the temperature of the support, pressure, and heating current $(3 \mathrm{~A}$, tungsten wire diameter $\approx 0.35 \mathrm{~mm}$ ) were kept constant during all experiments. The detailed gold film preparation procedure was described previously $[1,2]$.

\subsection{Thin Au film characterisation}

\subsubsection{Atomic force microscopy}

For atomic force microscopy studies we used a commercial instrument, model TMX 2000 "Discoverer" (TopoMetrix, CA), operating in contact (repulsive) and constant force modes under ambient conditions. Three scanners, $75 \mu \mathrm{m}, 25 \mu \mathrm{m}$, and $1 \mu \mathrm{m}$, and standard TopoMetrix $\mathrm{Si}_{3} \mathrm{~N}_{4}$ tips were applied. Samples were mounted on the scanners directly from the storage vessel. For all images we started from the same values of scan parameters (set point, rate, PID constants with $\mathrm{P}$ standing for proportional gain, I - for integral gain, and D - for derivative gain), however, in each case final optimisations were performed.

\subsubsection{Grazing incidence X-ray reflectometry}

The X-ray measurements were performed at grazing incidence geometry by means of a Philips high-resolution diffractometer equipped with a four-reflection 
$220 \mathrm{Ge}$ monochromator and a two-reflection $220 \mathrm{Ge}$ analyser. The characteristic $\mathrm{Cu} K_{\alpha 1}$ radiation $(\lambda=0.15406 \mathrm{~nm})$ was applied.

\section{Results}

\subsection{AFM investigations}

In dependence on the deposition time ranging from 2 to 50 min different kinds of thin gold films were obtained from the isolated gold islands to thick continuous films, respectively. For each film several images from the atomic force microscope at various positions were taken to gain better knowledge of the variations of local structures [3-6]. In Fig. 1 we present typical AFM results from a $300 \times 300 \mathrm{~nm}^{2}$
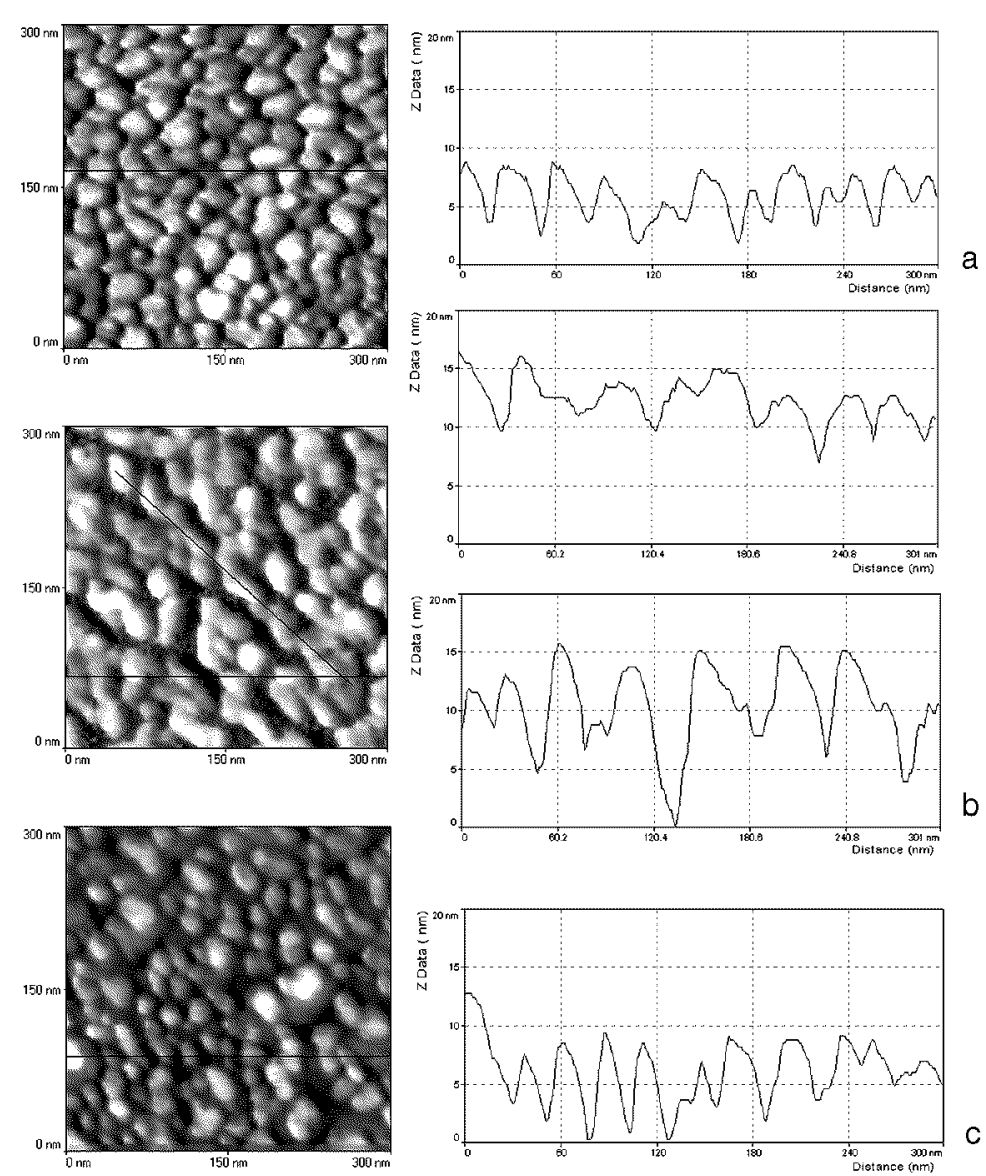

Fig. 1. AFM images (left) from a $300 \times 300 \mathrm{~nm}^{2}$ area of the thin Au films and surface profiles (right) corresponding to straight lines in the images. (a) Isolated Au nanometric islands (3 min deposition time), (b) very thin Au film consisting of chains of islands ( 8 min deposition time), and (c) continuous Au film (50 min deposition time). 
area for each kind of thin gold film. The AFM images (left) and surface profiles (right) demonstrate the differences in the surface nanostructure of the thin gold films of various thickness and colours.

During very short time deposition, shorter than 5 min, we obtained discontinuous thin Au films, consisting of isolated islands. In Fig. 1a the local structure of the transparent Au film of pale purple colour obtained in a 3 min evaporation (film A) is shown. The discontinuities within gold layer are visible as black spots surrounding the gold islands. The islands of gold composing the thin film are of similar diameter equal to $25 \mathrm{~nm}$, however, their height varies as a result of overlapping of several Au clusters. The presented profile indicates a nanometric structure of the film. The average height of the cluster approaches to $4 \mathrm{~nm}$ and the average thickness of Au film to $5.0 \mathrm{~nm}$. The average surface density of gold islands is $1.5 \times 10^{11} \mathrm{~cm}^{-2}$. The electrical resistance of discontinuous thin film measured in our deposition chamber was higher than $10^{8} \Omega$.

In Fig. 1b the AFM image for the transparent gold film of green colour obtained in $8 \mathrm{~min}$ is shown. Films B are also discontinuous, but they consist of the chains of islands of gold touching each other. For this film two different profiles are presented corresponding to the inclined straight line along the chain of islands (the upper one) and the level line along breadth of the AFM picture (the lower one), respectively. In this case the electrical resistance was higher than $10^{6} \Omega$.

As a result of a $50 \mathrm{~min}$ deposition the non-transparent $\mathrm{Au}$ films of golden colour were obtained. As one can see in Fig. 1c films $\mathrm{C}$ are continuous. Their resistance was equal to $\approx 2 \Omega$.

To determine the average root-mean-square (RMS) value of the surface roughness of the gold films studied, an area of $2000 \times 2000 \mathrm{~nm}^{2}$ was taken into account. The RMS values of the surface roughness were found to be equal to $1.6 \mathrm{~nm}$, $1.3 \mathrm{~nm}$, and $0.9 \mathrm{~nm}$ for film A, B, and C, respectively.

\subsection{GIXR research}

For testifying the RMS values of the surface roughness of the Au films obtained from AFM research the X-ray grazing incidence reflectometry was used. Figure 2 shows the measured reflectivity profiles for films presented above in Fig. 1. The shapes of these curves, dependent on the film thickness, as well as the slopes of the part above the critical angle are different.

The results of these investigations were interpreted basing on the Fresnel theory in the way described in previous paper [7], using the program GIXS [8] in distorted wave Born approximation (DWBA) [9-11]. By adjusting the theoretical reflectivity profiles to the measured curves the root-mean-square values of the surface roughness for different gold films were obtained. The fitting parameters are the following: the wavelength of incident radiation $\lambda=0.15406 \mathrm{~nm}$, the RMS value of the surface roughness of glass substrate $\sigma_{1}=0.5 \mathrm{~nm}$, the substrate density $\rho_{1}=2.65 \mathrm{~g} / \mathrm{cm}^{3}$, and the Au film density $\rho_{2}=19.28 \mathrm{~g} / \mathrm{cm}^{3}$. The RMS values of 


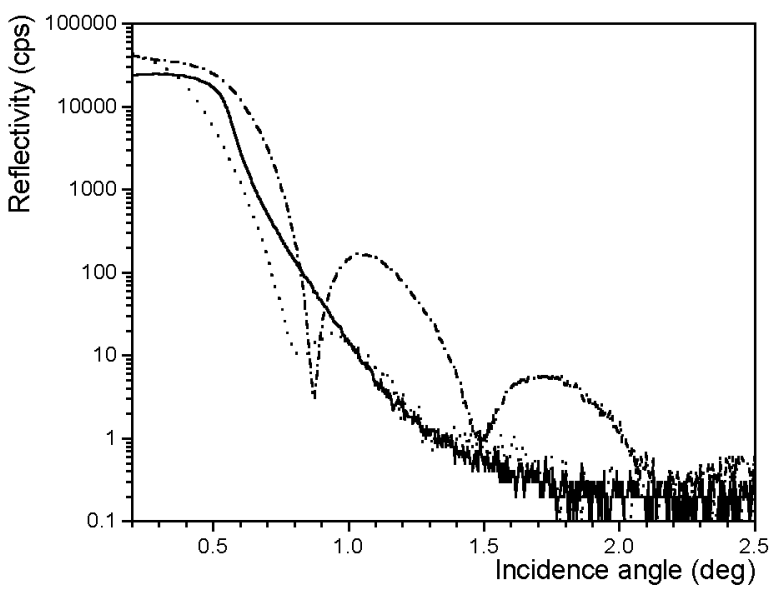

Fig. 2. Measured reflectivity profiles of the thin gold films: the dotted line for discontinuous film $\mathrm{A}$, the dashed-dotted line for the very thin film $\mathrm{B}$, and the solid line for continuous film $\mathrm{C}$.

the surface roughness were found to be equal to $1.2 \mathrm{~nm}, 1.0 \mathrm{~nm}$, and $0.7 \mathrm{~nm}$ for films $\mathrm{A}, \mathrm{B}$, and $\mathrm{C}$, respectively.

\section{Discussion}

Thin gold films deposited on glass were investigated by means of the mentioned above complementary methods, atomic force microscopy and X-ray reflectometry. A good agreement of the results was achieved, though each of methods bases on the different physical phenomenon. The GIXR determines the average surface roughness from large surfaces, at least of $1.0 \mathrm{~cm}^{2}$, and the AFM gives a local information concerning the surface morphology. It should be expected that a good accordance between X-ray and microscopic results could be obtained when for microscopic observations a large enough area is taken into account or the number of local observations is large enough allowing to get a mean value [12]. Therefore we chose the $2000 \times 2000 \mathrm{~nm}^{2}$ area for determination of the surface roughness by means of AFM. A comparison between the results of AFM and X-ray studies is given in Table.

A good agreement of the results obtained by both methods was found. As one can see, the RMS value of the surface roughness of the Au film decreases when the film thickness increases.

\section{Conclusions}

The aim of the present paper was the study of thin gold films obtained by UHV evaporation at low temperatures on glass substrates as a function of the 
TABLE

The surface roughness of thin gold films.

\begin{tabular}{c|c|c|c|c}
\hline \hline Film & $\begin{array}{c}\text { Deposition time } \\
{[\mathrm{min}]}\end{array}$ & $\begin{array}{c}\text { Film thickness } \\
{[\mathrm{nm}]}\end{array}$ & $\begin{array}{c}\text { AFM RMS } \\
\text { surface roughness } \\
{[\mathrm{nm}]}\end{array}$ & $\begin{array}{c}\text { GIXR RMS } \\
\text { surface roughness } \\
{[\mathrm{nm}]}\end{array}$ \\
\hline A & 3 & 5 & 1.6 & 1.2 \\
B & 8 & 20 & 1.3 & 1.0 \\
C & 50 & 50 & 0.9 & 0.7
\end{tabular}

time of the process. For the research the atomic force microscopy and the grazing incidence X-ray reflectometry were used. The AFM method shows the morphology of the investigated gold films, whereas GIXR gives average information about the real film surface. It was confirmed that the evaporation process goes through a number of distinct stages. The gold-gold interaction is stronger in comparison to the local interaction of gold with the glass substrate. Due to the deposition duration different kinds of thin gold films were obtained.

The electrical properties of thin gold films depend on their structure. The discontinuous films of island structure exhibit high electrical resistance because the metallic islands do not touch each other, while the continuous films are characterised by metallic behaviour. Thus, the colour of thin gold films, their electrical resistance and nanostructure characterise the stages of the film growing.

\section{References}

[1] R. Nowakowski, T. Kobiela, Z. Wolfram, R. Duś, Appl. Surf. Sci. 115, 217 (1997).

[2] L. Stobiński, L. Zommer, R. Duś, Appl. Surf. Sci. 141, 319 (1999).

[3] S.E. Roark, K.L. Rowlen, Anal. Chem. 60, 261 (1994).

[4] D.J. Semin, K.L. Rowlen, Anal. Chem. 66, 4324 (1994).

[5] X.M. Yang, K. Ajito, D.A. Tryk, K. Hashimoto, A. Fujishima, J. Phys. Chem. 100, 7293 (1996).

[6] S.J. Fang, W. Chen, T. Yamanaka, R.C. Helms, Appl. Phys. Lett. 68, 2837 (1996).

[7] D. Żymierska, E. Sobczak, K. Godwod, S. Miotkowska, in: Proc. XVII Conf. on Applied Crystallography, Eds. H. Morawiec, D. Stróż, World Sci., Singapore 1998, p. 394.

[8] M. Wormington, GIXS (Grazing Incidence X-ray Scattering) Software, Bede Scientific Instruments Ltd. and University of Warwick, UK.

[9] L.G. Parratt, Phys. Rev. 95, 359 (1954).

[10] S.K. Sinha, E.B. Sirota, S. Garoff, H.B. Stanley, Phys. Rev. B 38, 2297 (1988).

[11] D.K. Bowen, B.K. Tanner, Nanotechnology 4, 175 (1993).

[12] D. Żymierska, J. Auleytner, T. Kobiela, R. Duś, Phys. Status Solidi A 180, 479 (2000). 